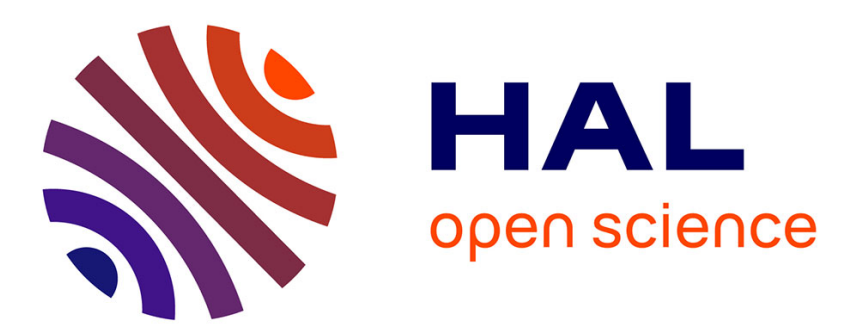

\title{
Shell finite element based on the Proper Generalized Decomposition for the modeling of cylindrical composite structures.
}

\author{
P. Vidal, L. Gallimard, O. Polit
}

\section{- To cite this version:}

P. Vidal, L. Gallimard, O. Polit. Shell finite element based on the Proper Generalized Decomposition for the modeling of cylindrical composite structures.. Computers \& Structures, 2014, 132, pp.1-11. 10.1016/j.compstruc.2013.10.015 . hal-01366963

\section{HAL Id: hal-01366963 https://hal.science/hal-01366963}

Submitted on 28 Oct 2019

HAL is a multi-disciplinary open access archive for the deposit and dissemination of scientific research documents, whether they are published or not. The documents may come from teaching and research institutions in France or abroad, or from public or private research centers.
L'archive ouverte pluridisciplinaire HAL, est destinée au dépôt et à la diffusion de documents scientifiques de niveau recherche, publiés ou non, émanant des établissements d'enseignement et de recherche français ou étrangers, des laboratoires publics ou privés. 


\title{
Shell finite element based on the Proper Generalized Decomposition for the modeling of cylindrical composite structures
}

\author{
P. Vidal *, L. Gallimard, O. Polit \\ Laboratoire Energétique, Mécanique, Electromagnétisme, Université Paris Ouest Nanterre-La Défense, 50 rue de Sèvres, 92410 Ville d'Avray, France
}

A B S T R A C T

Keywords:

Composite structures

Cylindrical shell

Finite element analysis

Proper Generalized Decomposition
The introduction of the Proper Generalized Decomposition (PGD) is presented for the layer-wise modeling of heterogeneous cylindrical shells. The displacement field is approximated as a sum of separated functions of the in-plane coordinates and the transverse coordinate. This choice yields to an iterative process that consists of solving a 2D and 1D problem successively at each iteration. In the thickness direction, a fourth-order expansion in each layer is considered. For the in-plane description, classical Finite Element method is used. The approach is assessed through mechanical tests for thin/thick and deep/shallow laminated cylindrical shells. Both convergence rate and accuracy are discussed.

\section{Introduction}

Composite shells are widely used in the industrial field (aerospace, automotive, marine, medical industries...) due to their excellent mechanical properties, especially their high specific stiffness and strength. For composite design, accurate knowledge of displacements and stresses is required. One way consists in considering three-dimensional modelisation. However, due to the complexity of such numerical simulations, it is suitable to represent the problem as a two-dimensional model leading to the construction of shell theories. There are two ways for defining the approximation of the displacement field. A "pure shell model" can be considered in which the displacement is associated with the local curvilinear vectors, and strain and stress are deduced using differential geometry [1]. Alternatively, the shell-like solid approach [2] for obtaining shell FE is widely used in commercial software, as it is more simple. In this case, the displacement vector is defined in the global cartesian frame and jacobian matrix transformation is used to express strain and stress with respect to reference frame defined on the middle surface in order to introduce the constitutive law. In this approach, differentiation is simplified and the curvatures are not directly calculated [3]. So, the development of efficient computational models for the analysis of shells appears thus of major interest.

According to published research, various theories based on the Finite Element (FE) method for composite shells have been developed. In the following, most of the mentioned works refer to the pure shell model. Thus, two families of models [4] can be identified:

\footnotetext{
* Corresponding author. Tel.: +33 140974855.

E-mail address: philippe.vidal@u-paris10.fr (P. Vidal).
}

- the Equivalent Single Layer Models (ESLM), where the classical Shell Theory (CST/Koïter) and First Order Shear Deformation Theory (FSDT/Nagdhi) models can be found. The reader can refer to [9] to have a description of the assumptions on the strain for deriving different shell models. CST leads to inaccurate results for composites because both transverse shear and normal strains are neglected. Triangular and rectangular elements are used in [5-7] respectively, for shallow laminated shells. FSDT is the most popular model due to the possibility to use a $\mathrm{C}^{0} \mathrm{FE}$, but it needs shear correction factors and transverse normal strain is always neglected. A rectangular isoparametric element based on a MITC approach is presented in a recent work [8]. So, Higher-order Shear Deformation Theories (HSDT) have been developed to overcome these drawbacks. Sgambitterra et al. [10] proposes a three-node flat shell element based on a 1,2-order theory including 7 parameters. A HSDT with 9 parameters based on a nine-node quadrilateral isoparametric element is developed by Kant and Menon [11]. A third-order theory with a four-node isoparametric element including 7 parameters is considered in [12]. Different theories based on the Carrera's Unified Formulation are addressed in [13].

In the ESLM context, a simple way to improve the estimation of the mechanical quantities consists in adding one zig-zag function (Murakami) in the expression of the displacement to introduce the slope discontinuity at the interface between two adjacent layers. It allows to describe the so-called zig-zag effect. It has been carried out by Brank [14]. The approach includes 7 parameters and is based on the Reissner's formulation. See also the work of Bhaskar [15] based on a HSDT approach. 
- the Layer-Wise Models (LWM) where the expression of the mechanical quantities is written in each layer. A quadratic triangle element based on a constant shear angle is considered in [16], but a shear correction factor is needed. A three-dimensional shell element is proposed in [17]. A LW triangle FE is developed in [18] with a condensation technique at the pre-processing level. [19] deals with a hybrid strain flat triangular FE based on the Hellinger-Reissner variational principle. Note that the transverse normal and shear stresses are only taken into account in [20] where a four-node isoparametric assumed strain is considered. We can mention the eight-node 3D hybrid-EAS solid shell element based on the Hu-Washizu variational principle in [21]. See also the previously mentioned work [13]. In all the aforementioned works, the number of unknowns depends on the number of layers.

As an alternative, refined models have been developed in order to improve the accuracy of ESL models avoiding the additional computational cost of LW approach. Based on physical considerations and after some algebraic transformations, the number of unknowns becomes independent of the number of layers. We can mention the recent work of Yasin [22] dedicated to shallow shells. A four-node quadrilateral element with 5 parameters is built. Shariyat [23] has also developed a so-called zig-zag model including 15 parameters. A full compatible Hermitian rectangular elements are employed. It should be also mentioned the work of Dau [1] where a $C^{1}$ triangular six-node FE (Argyris-Ganev) based on the Sinus model is considered involving 5 parameters. The approach ensures the continuity conditions of the transverse shear stresses at the interfaces between two adjacent layers.

For the present topics, it should be noted that the mentioned works are based on the Finite Element method for linear elasticity problem in mechanics and applied to laminated composites, knowing that many other approaches (meshless, analytical, semi-analytical...) are involved in open literature. Furthermore, the fundamental subject about the shear and membrane locking of shell is not addressed here. So, this above literature deals with only some aspects of the broad research activity about composite shells. An extensive assessment of different approaches for both various theories and/or finite element applications can be found in [24-31].

Over the past years, the Proper Generalized Decomposition (PGD) [32-35] has shown interesting features in the reduction model framework. It has been used in the context of separation of coordinate variables in multi-dimensional PDEs [34]. And in particular, it has been applied for composite beams and plates in [36-40].

The main goal of this work consists in assessing the Proper Generalized Decomposition to model cylindrical composite shell structures. So, the present approach is based on the separation representation where the displacements are written under the form of a sum of products of bidimensional polynomials of $\left(\xi^{1}, \xi^{2}\right)$ and unidimensional polynomials of $z$. A piecewise fourth-order Lagrange polynomial of $z$ is chosen. As far as the variation with respect to the in-plane coordinates is concerned, a 2D eight-node quadrilateral FE is employed. Using the PGD, each unknown function of $\left(\xi^{1}, \xi^{2}\right)$ is classically approximated using one degree of freedom (dof) per node of the mesh and the LW unknown functions of $z$ are global for the whole shell. Finally, the deduced non-linear problem implies the resolution of two linear problems alternatively. This process yields to a $2 \mathrm{D}$ and a $1 \mathrm{D}$ problems in which the number of unknowns is smaller than a classical Layerwise approach. The interesting feature of this approach lies on the possibility to have a higher-order $z$-expansion and to refine the description of the mechanical quantities through the thickness without increasing the computational cost. This is particularly suitable for the modeling of composite structures.

We now outline the remainder of this article. First, the shell definition and the differential geometry are recalled. Then, the mechanical formulation is given. The principles of the PGD are precised in the framework of our study. The particular assumption on the displacements yields a non-linear problem and an iterative process is chosen to solve this one. The FE discretization is also described and finally, numerical tests are performed. A preliminary convergence study is performed. Then, the influence of classical assumptions on the strains and the number of numerical layers are studied. The approach is also assessed for deep and shallow shells and different slenderness ratios. The accuracy of the results is evaluated by comparison with a $2 \mathrm{D}$ elasticity solution from [41].

\section{Shell definitions and differential geometry}

A shell $\mathcal{C}$ with a middle surface $\mathcal{S}$ and a constant thickness $e$, see Fig. 1, is defined by Bernadou [42]:

$\mathcal{C}=\left\{M \in \mathcal{R}^{3}: \overrightarrow{O M}\left(\xi, \xi^{3}=z\right)=\vec{\Phi}(\xi)+z \vec{a}_{3} ; \xi \in \Omega ;-\frac{1}{2} e \leqslant z \leqslant \frac{1}{2} e\right\}$

where the middle surface can be described by a map $\vec{\Phi}$ from a parametric bidimensional domain $\Omega$ as:

$$
\begin{array}{r}
\vec{\Phi}: \quad \Omega \subset \mathcal{R}^{2} \rightarrow \mathcal{S} \subset \mathcal{R}^{3} \\
\xi=\left(\xi^{1}, \xi^{2}\right) \mapsto \vec{\Phi}(\xi)
\end{array}
$$

In Fig. 1, the map $\vec{\Phi}$ describing the shell middle surface (in grey) and the local basis vectors are presented. The basis vectors $\vec{a}_{i}$ are defined for a point on $\mathcal{S}$ and the basis vectors $\vec{g}_{i}$ are defined for a generic point of the shell.

For a point on the shell middle surface, the covariant basis vectors defining the tangent plane to the middle surface are usually obtained as follows:

$\vec{a}_{\alpha}=\vec{\Phi}\left(\xi^{1}, \xi^{2}\right)_{, \alpha} ; \quad \vec{a}_{3}=\frac{\vec{a}_{1} \times \vec{a}_{2}}{\left\|\vec{a}_{1} \times \vec{a}_{2}\right\|}$

where $\vec{a}_{3}$ is the unit normal vector to the surface $\mathcal{S}$, see Fig. 1. In Eq. (2) and further on, latin indices $i, j, \ldots$ take their values in the set $\{1,2,3\}$ while greek indices $\alpha, \beta, \ldots$ take their values in the set $\{1,2\}$. The summation convention on repeated indices is used and partial derivative is denoted by ()$_{,}$.

A shell is characterized by the first fundamental form $a_{\alpha \beta}$ and the second one $b_{\alpha \beta}$. Their covariant, contravariant and mixed form definitions are given by:

$a_{\alpha \beta}=\vec{a}_{\alpha} \cdot \vec{a}_{\beta} \quad a^{\alpha \beta}=\vec{a}^{\alpha} \cdot \vec{a}^{\beta} \quad b_{\alpha \beta}=\vec{a}_{\alpha \alpha, \beta} \cdot \vec{a}_{3} \quad b_{\alpha}^{\beta}=\vec{a}^{\beta} \cdot \vec{a}_{3, \alpha}$

For a generic point of the shell, covariant basis vectors must be defined and we have:

$\vec{g}_{\alpha}=\overrightarrow{O M}(\xi, z)_{, \alpha}=\left(\delta_{\alpha}^{\beta}-z b_{\alpha}^{\beta}\right) \vec{a}_{\beta}=\mu_{\alpha}^{\beta}(z) \vec{a}_{\beta}$ and $\vec{g}_{3}=\vec{a}_{3}$

where $\delta_{\alpha}^{\beta}$ is the Kronecker symbol and $b_{\alpha}^{\beta}$ is the mixed form of the second fundamental form. This basis $\vec{g}_{i}$, illustrated in Fig. 1, must be used to define quantities for any point of the shell. The form $\mu_{\alpha}^{\beta}(z)$ introduced in Eq. (4) defines the transport from the shell middle surface to any point of the shell and is associated with the curvature variation along the thickness direction $z$ of the shell. The inverse tensor of the mixed tensor $\mu_{\alpha}^{\beta}$ is denoted $m_{\alpha}^{\beta}$ and is defined as:

$m_{\alpha}^{\beta}=\left(\mu^{-1}\right)_{\alpha}^{\beta}=\frac{1}{\mu}\left\{\delta_{\alpha}^{\beta}+z\left(b_{\alpha}^{\beta}-2 H \delta_{\alpha}^{\beta}\right)\right\}$ 


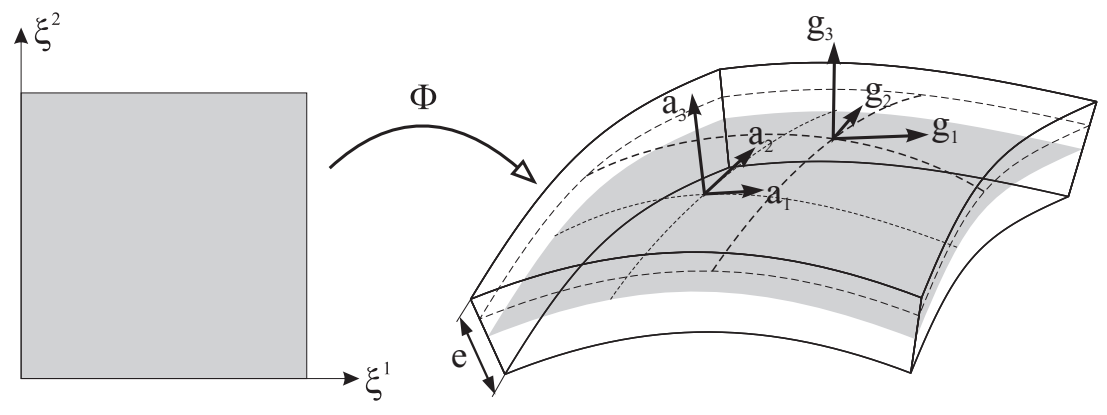

Fig. 1. The map $\vec{\Phi}$ and the local basis vectors $\vec{a}_{i}$ and $\vec{g}_{i}$ for a shell panel.

where we have introduced the determinant of the mixed tensor $\mu=\operatorname{det}\left(\mu_{\alpha}^{\beta}\right)=1-2 H \xi^{3}+\left(\xi^{3}\right)^{2} K$ and the invariants of the second fundamental form $H=\frac{1}{2} \operatorname{tr}\left(b_{\alpha}^{\beta}\right)$ and $K=\operatorname{det}\left(b_{\alpha}^{\beta}\right)$. Finally, the surface element $d \mathcal{S}$ and the volume element $d \mathcal{C}$ are given by:

$d \mathcal{S}=\sqrt{a} d \xi^{1} d \xi^{2} \quad d \mathcal{C}=\mu d \mathcal{S} d z$

where $a$ is the determinant of the first fundamental form $a_{\alpha \beta}$. The geometry of a shell can also be defined using contravariant or mixed forms. Furthermore, covariant and contravariant differentiation involving Christoffel symbols are not detailed here and readers can refer to the book [42].

\section{Reference problem description}

\subsection{The definition of the strain field}

For geometrically linear elastic analysis, the components of the strain tensor $\varepsilon_{i j}$ expressed in the contravariant basis $\vec{a}^{i}$ are obtained through covariant differentiation, denoted |, as follows:

$$
\begin{aligned}
\varepsilon= & \varepsilon_{i j}\left(\vec{a}^{i} \otimes \vec{a}^{j}\right) \text { with } \\
& 2 \varepsilon_{\gamma \lambda}=m_{\lambda}^{\beta}\left(u_{\gamma \mid \beta}-b_{\gamma \beta} u_{3}\right)+m_{\gamma}^{\alpha}\left(u_{\lambda \mid \alpha}-b_{\lambda \alpha} u_{3}\right) \\
& 2 \varepsilon_{\gamma 3}=u_{\gamma \mid 3}+m_{\gamma}^{\alpha}\left(u_{3, \alpha}+b_{\alpha}^{\lambda} u_{\lambda}\right) \\
& \varepsilon_{33}=u_{3,3}
\end{aligned}
$$

The mixed tensor $m_{\lambda}^{\beta}$ carries out the transport from any point of the shell to the shell middle surface, that is from $\vec{g}^{i}$ to $\vec{a}^{i}$.

\subsection{Constitutive relation}

The stress tensor is obtained from the strain tensor using the constitutive equations. For this purpose, all these tensors must be referred to the covariant and contravariant basis vectors, $\vec{a}_{i}$ and $\vec{a}^{i}$ respectively, associated with the middle surface of the shell. In case of laminated shells composed of orthotropic plies, this reference frame ensures to consistently take into account the different material orientations of the layers. The tensorial relation is:

$\sigma^{i j}=C^{i j k l} \varepsilon_{k l}$ with $\sigma^{i j}\left(\vec{a}_{i} \otimes \vec{a}_{j}\right), \quad C^{i j k l}\left(\vec{a}_{i} \otimes \vec{a}_{j} \otimes \vec{a}_{k} \otimes \vec{a}_{l}\right), \quad \varepsilon_{k l}\left(\vec{a}^{k} \otimes \vec{a}^{l}\right)$

It should be noted that the stress tensor is defined in the covariant reference frame, whereas the strain components are defined in the contravariant frame. If the frame is assumed to be orthonormal then covariant and contravariant components are equal, that is superscript and sub-script are interchangeable. With this assumption and for a shell made of $N C$ perfectly bonded orthotropic layers, the three-dimensional constitutive law of the $k$ th layer is expressed in matrix notation by:

$$
\left[\begin{array}{c}
\sigma_{11}^{(k)}(\xi, z) \\
\sigma_{22}^{(k)}(\xi, z) \\
\sigma_{33}^{(k)}(\xi, z) \\
\sigma_{23}^{(k)}(\xi, z) \\
\sigma_{13}^{(k)}(\xi, z) \\
\sigma_{12}^{(k)}(\xi, z)
\end{array}\right]=\left[\begin{array}{cccccc}
C_{11}^{(k)} & C_{12}^{(k)} & C_{13}^{(k)} & 0 & 0 & 0 \\
& C_{22}^{(k)} & C_{23}^{(k)} & 0 & 0 & 0 \\
& & C_{33}^{(k)} & 0 & 0 & 0 \\
& & & C_{44}^{(k)} & 0 & 0 \\
& & & & C_{55}^{(k)} & 0 \\
& & & & & C_{66}^{(k)}
\end{array}\right]\left[\begin{array}{c}
\varepsilon_{11}^{(k)}(\xi, z) \\
\varepsilon_{22}^{(k)}(\xi, z) \\
\varepsilon_{33}^{(k)}(\xi, z) \\
\gamma_{23}^{(k)}(\xi, z) \\
\gamma_{13}^{(k)}(\xi, z) \\
\gamma_{12}^{(k)}(\xi, z)
\end{array}\right]
$$

where we denote $C_{i j}^{(k)}$ the three-dimensional stiffness coefficients of the layer $k$, the stress vector $[\sigma]$ and the strain vector $[\varepsilon]$.

\subsection{The weak form of the boundary value problem}

Using the above matrix notation and for admissible displacement $\delta \vec{u} \in \delta U$, the variational principle is given by:

Find $\vec{u} \in U$ (space of admissible displacements) such that

$-\int_{\mathcal{C}}[\varepsilon(\delta \vec{u})]^{T}[\sigma(\vec{u})] d \mathcal{C}+\int_{\mathcal{C}}[\delta u]^{T}[b] d \mathcal{C}+\int_{\partial \mathcal{C}_{F}}[\delta u]^{T}[t] d \partial \mathcal{C}$

$=0, \quad \forall \delta \vec{u} \in \delta U$

where $[\mathrm{b}]$ and $[\mathrm{t}]$ are the prescribed body and surface forces applied on $\partial \mathcal{C}_{F}$.

\section{Application of the Proper Generalized Decomposition to cylindrical shell}

In this section, we develop the application of the PGD for shell analysis. This work is an extension of the previous studies on beam and plate structures [38-40].

\subsection{Cylindrical geometry}

A cylindrical shell (see Fig. 2) is described using the following map:

$\left\{\begin{array}{l}x_{1}\left(\xi^{1}, \xi^{2}\right)=R \cos \left(\frac{\xi^{1}}{R}\right) \\ x_{2}\left(\xi^{1}, \xi^{2}\right)=R \sin \left(\frac{\xi^{1}}{R}\right) \\ x_{3}\left(\xi^{1}, \xi^{2}\right)=\xi^{2}\end{array}\right.$

Following Eq. (3), the non-zero terms for the covariant and mixed forms are:

$$
\begin{aligned}
a_{11} & =a_{22}=1 \quad b_{11}=b_{1}^{1}=-\frac{1}{R} \quad \mu_{1}^{1}=1+\frac{z}{R} \quad m_{1}^{1} \\
& =\left(1+\frac{z}{R}\right)^{-1}
\end{aligned}
$$

and $\mu=1+\frac{z}{R}$ 


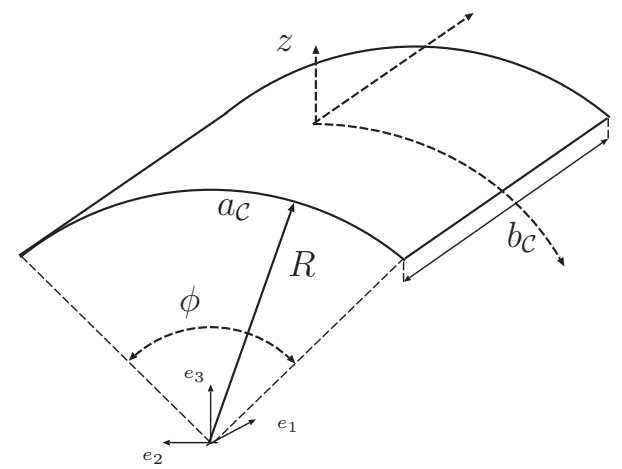

Fig. 2. Middle surface of the cylindrical shell.

\subsection{The displacement}

Let us denote $\left(u_{1}\left(\xi^{1}, \xi^{2}, \xi^{3}=z\right), u_{2}\left(\xi^{1}, \xi^{2}, \xi^{3}=z\right), u_{3}\left(\xi^{1}, \xi^{2}, \xi^{3}=z\right)\right)$ the curvilinear components of the displacement field associated with the contravariant basis vectors $\vec{a}^{i}$. Let $\Omega$ and $\Omega_{z}$ the bidimensional domain associated with the mid-surface of the shell (see Eq. (1)), and the unidimensional domain associated with the normal fiber respectively. This displacement solution is constructed as the sum of $N$ products of functions of in-plane coordinates and transverse coordinate $(N \in \mathbb{N}$ is the order of the representation)

$[u]=\left[\begin{array}{l}u_{1}\left(\xi, \xi^{3}=z\right) \\ u_{2}\left(\xi, \xi^{3}=z\right) \\ u_{3}\left(\xi, \xi^{3}=z\right)\end{array}\right]=\sum_{j=1}^{N}\left[\begin{array}{l}f_{1}^{j}(z) v_{1}^{j}(\xi) \\ f_{2}^{j}(z) v_{2}^{j}(\xi) \\ f_{3}^{j}(z) v_{3}^{j}(\xi)\end{array}\right]$

where $\left(f_{1}^{j}, f_{2}^{j}, f_{3}^{j}\right)$ are defined in $\Omega_{z}$ and $\left(v_{1}^{j}, v_{2}^{j}, v_{3}^{j}\right)$ are defined in $\Omega$. In this paper, a classical eight-node FE approximation is used in $\Omega$ and a LW description is chosen in $\Omega_{z}$ as it is particulary suitable for the modeling of composite structure.

\subsection{The strain field for the cylindrical composite structure}

The strain field in Eq. (7) is free of any approximated shell kinematics. These strain components are simplified using Eq. (12) and we recover the following relations:

$$
\begin{aligned}
& \varepsilon_{11}=\left(1+\frac{z}{R}\right)^{-1}\left(u_{1,1}+\frac{1}{R} u_{3}\right) \\
& \varepsilon_{22}=u_{2,2} \\
& \varepsilon_{33}=u_{3,3} \\
& \gamma_{23}=u_{2,3}+u_{3,2} \\
& \gamma_{13}=u_{1,3}+\left(1+\frac{z}{R}\right)^{-1}\left(u_{3,1}-\frac{1}{R} u_{1}\right) \\
& \gamma_{12}=u_{1,2}+\left(1+\frac{z}{R}\right)^{-1} u_{2,1}
\end{aligned}
$$

where covariant derivative becomes classical derivative for the case of a cylindrical shell.

Eq. (13) must be introduced at this level in order to obtain the compatible strain expansion along the normal coordinate $z$ of the shell. So, we obtain:

$$
[\varepsilon(u)]=\sum_{j=1}^{N}\left[\begin{array}{c}
\mu^{-1}\left(f_{1}^{j} v_{1,1}^{j}+\frac{1}{R} f_{3}^{j} v_{3}^{j}\right) \\
f_{2}^{j} v_{2,2}^{j} \\
\left(f_{3}^{j}\right)^{\prime} v_{3}^{j} \\
\left(f_{2}^{j}\right)^{\prime} v_{2}^{j}+f_{3}^{j} v_{3,2}^{j} \\
\left(f_{1}^{j}\right)^{\prime} v_{1}^{j}+\mu^{-1}\left(f_{3}^{j} v_{3,1}^{j}-\frac{1}{R} f_{1}^{j} v_{1}^{j}\right) \\
f_{1}^{j} v_{1,2}^{j}+\mu^{-1} f_{2}^{j} v_{2,1}^{j}
\end{array}\right]
$$

where the prime stands for the classical derivative $\left(f_{i}^{\prime}=\frac{d f_{i}}{d z}\right),()_{, \alpha}$ for the partial derivative and $\mu=1+\frac{z}{R}$.

\subsection{The problem to be solved}

For sake of clarity, the body forces are neglected in the developments and the weak form of the shell problem introduced in Eq (10) simplifies in

$\int_{\mathcal{S}} \int_{\Omega_{z}}[\varepsilon(\delta \vec{u})]^{T}[C][\varepsilon(\vec{u})] \mu d z d \mathcal{S}+\int_{\partial \mathcal{C}_{F}}[\delta u]^{T}[t] d \partial \mathcal{C}=0$

where $[C]$ represents, in each layer $(k)$, the matrix of the elastic moduli. For the present work, $\partial \mathcal{C}_{F}$ is considered as the top or bottom surface of the shell, that is $z=z_{F}$ with $z_{F}= \pm e / 2$.

Eq. (16) is solved by an iterative procedure. If we assume that the first $n$ functions have been already computed, the trial function for the iteration $n+1$ is written as

$\left[u^{n+1}\right]=\left[u^{n}\right]+\left[\begin{array}{ll}f_{1} & v_{1} \\ f_{2} & v_{2} \\ f_{3} & v_{3}\end{array}\right]$

where $\left(v_{1}, v_{2}, v_{3}\right),\left(f_{1}, f_{2}, f_{3}\right)$ are the functions to be computed and $\left[u^{n}\right]$ is the associated known set at iteration $n$ defined by

$\left[u^{n}\right]=\sum_{i=1}^{n}\left[\begin{array}{l}f_{1}^{i} v_{1}^{i} \\ f_{2}^{i} v_{2}^{i} \\ f_{3}^{i} v_{3}^{i}\end{array}\right]$

The test function is

$\delta\left[\begin{array}{ll}f_{1} & v_{1} \\ f_{2} & v_{2} \\ f_{3} & v_{3}\end{array}\right]=\left[\begin{array}{l}\delta f_{1} v_{1}+f_{1} \delta v_{1} \\ \delta f_{2} v_{2}+f_{2} \delta v_{2} \\ \delta f_{3} v_{3}+f_{3} \delta v_{3}\end{array}\right]=[V][\delta f]+[F][\delta v]$

with

$[v]=\left[\begin{array}{l}v_{1} \\ v_{2} \\ v_{3}\end{array}\right] \quad[f]=\left[\begin{array}{l}f_{1} \\ f_{2} \\ f_{3}\end{array}\right] \quad[V]=\left[\begin{array}{ccc}v_{1} & 0 & 0 \\ 0 & v_{2} & 0 \\ 0 & 0 & v_{3}\end{array}\right] \quad[F]=\left[\begin{array}{ccc}f_{1} & 0 & 0 \\ 0 & f_{2} & 0 \\ 0 & 0 & f_{3}\end{array}\right]$

The test function defined by Eq. (19) and the trial function defined by Eq. (17) are introduced into the weak form Eq. (16) to obtain the two following equations:

$$
\begin{aligned}
& \int_{\mathcal{S}} \int_{\Omega_{z}}\left([\varepsilon(F \delta v)]^{T}[C][\varepsilon(F v)]\right) \mu d z d \mathcal{S} \\
& =\int_{\mathcal{S}} \int_{\Omega_{z}}-[\varepsilon(F \delta v)]^{T}[C]\left[\varepsilon\left(u^{n}\right)\right] \mu d z d \mathcal{S} \\
& \quad+\left.\int_{\mathcal{S}}\left([F \delta v]^{T}[t] \mu\right)\right|_{z=z_{F}} d \mathcal{S} \\
& \int_{\Omega_{z}} \int_{\mathcal{S}}\left([\varepsilon(V \delta f)]^{T}[C][\varepsilon(V f)]\right) d \mathcal{S} \mu d z \\
& =\int_{\Omega_{z}} \int_{\mathcal{S}}-[\varepsilon(V \delta f)]^{T}[C]\left[\varepsilon\left(u^{n}\right)\right] d \mathcal{S} \mu d z+\left.\int_{\mathcal{S}}[V \delta f]^{T}[t] \mu d \mathcal{S}\right|_{z=z_{F}}
\end{aligned}
$$

As these equations define a coupled non linear problem, a non linear resolution strategy has to be used. The simplest strategy is a fixed point method. An initial function $f^{(0)}$ is set, and at each step, the algorithm computes a new pair $\left(v^{(m+1)}, f^{(m+1)}\right)$ such that

- $v^{(m+1)}$ satisfies Eq. (21) for $f$ set to $f^{(m)}$ (i.e. $F$ is given)

- $f^{(m+1)}$ satisfies Eq. (22) for $v$ set to $v^{(m+1)}$ (i.e. $V$ is given)

These two equations are linear and the first one is solved on $\mathcal{S}$, while the second one is solved on $\Omega_{z}$. As in [40], the fixed point 
algorithm is stopped when the relative distance $\varepsilon$ between two consecutive solutions is smaller than a fixed value $\varepsilon_{0}$. In this paper, we use $\varepsilon_{0}=10^{-3}$.

\subsection{Finite element discretization}

To build the shell finite element approximation, a discrete representation of the functions $(v, f)$ must be introduced. We use a classical finite element approximation in $\Omega$, and a polynomial expansion in $\Omega_{z}$. The elementary vector of degrees of freedom (dof) associated with one element $\Omega_{e}$ of the mesh in $\Omega$ is denoted $\left[q_{e}^{v}\right]$. The vector of dofs associated with the polynomial expansion in $\Omega_{z}$ is denoted $\left[q^{f}\right]$. The displacement fields and the strain fields are determined from the values of $\left[q_{e}^{v}\right]$ and $\left[q^{f}\right]$ by

$$
\begin{aligned}
{\left[v_{e}\right] } & =\left[N_{\xi}\right]\left[q_{e}^{v}\right], \quad\left[\mathcal{E}_{v}^{e}\right]=\left[B_{\xi}\right]\left[q_{e}^{v}\right], \quad[f]=\left[N_{z}\right]\left[q^{f}\right] \text { and }\left[\mathcal{E}_{f}\right] \\
& =\left[B_{z}\right]\left[q^{f}\right]
\end{aligned}
$$

where

$\left[\mathcal{E}_{v}^{e}\right]^{T}=\left[\begin{array}{lllllllll}v_{1} & v_{1,1} & v_{1,2} & v_{2} & v_{2,1} & v_{2,2} & v_{3} & v_{3,1} & v_{3,2}\end{array}\right]$

and

$\left[\mathcal{E}_{f}\right]^{T}=\left[\begin{array}{llllll}f_{1} & f_{1}^{\prime} & f_{2} & f_{2}^{\prime} & f_{3} & f_{3}^{\prime}\end{array}\right]$

The matrices $\left[N_{\xi}\right],\left[B_{\xi}\right],\left[N_{z}\right],\left[B_{z}\right]$ contain the interpolation functions, their derivatives and the jacobian components.

\subsection{Finite element problem to be solved on $\Omega$}

For the sake of simplicity, the function $f^{(m)}$ which is assumed to be known, will be denoted $\tilde{f}$ (and $\tilde{F}$ ), and the function $v^{(m+1)}$ to be computed will be denoted $v$. The strain in Eq. (21) is defined in matrix notations as

$[\varepsilon(\tilde{F} v)]=\left[\Sigma_{z}(\tilde{f})\right]\left[\mathcal{E}_{v}\right]$

with

$$
\left[\Sigma_{z}(\tilde{f})\right]=\left[\begin{array}{ccccccccc}
0 & \tilde{f}_{1} / \mu & 0 & 0 & 0 & 0 & \tilde{f}_{3} /(\mu R) & 0 & 0 \\
0 & 0 & 0 & 0 & 0 & \tilde{f}_{2} & 0 & 0 & 0 \\
0 & 0 & 0 & 0 & 0 & 0 & \tilde{f}_{3}^{\prime} & 0 & 0 \\
0 & 0 & 0 & \tilde{f}_{2}^{\prime} & 0 & 0 & 0 & 0 & \tilde{f}_{3} \\
\tilde{f}_{1}^{\prime}-\tilde{f}_{1} /(\mu R) & 0 & 0 & 0 & 0 & 0 & 0 & \tilde{f}_{3} / \mu & 0 \\
0 & 0 & \tilde{f}_{1} & 0 & \tilde{f}_{2} / \mu & 0 & 0 & 0 & 0
\end{array}\right]
$$

The variational problem defined on $\Omega$ from Eqs. (21) and (6) is

$$
\begin{aligned}
\int_{\Omega}\left[\delta \mathcal{E}_{v}\right]^{T}\left[k_{z}(\tilde{f})\right]\left[\mathcal{E}_{v}\right] \sqrt{a} d \Omega= & \int_{\Omega}[\delta v]^{T}\left[t_{z}(\tilde{f})\right] \sqrt{a} d \Omega \\
& -\int_{\Omega}\left[\delta \mathcal{E}_{v}\right]^{T}\left[\sigma_{z}\left(\tilde{f}, u^{n}\right)\right] \sqrt{a} d \Omega
\end{aligned}
$$

with

$\left[k_{z}(\tilde{f})\right]=\int_{\Omega_{z}}\left[\Sigma_{z}(\tilde{f})\right]^{T}[C]\left[\Sigma_{z}(\tilde{f})\right] \mu d z$

$\left[t_{z}(\tilde{f})\right]=\left.\left([\tilde{F}]^{T}[t] \mu\right)\right|_{z=z_{F}}$

$\left[\sigma_{z}\left(\tilde{f}, u^{n}\right)\right]=\int_{\Omega_{z}}\left[\Sigma_{z}(\tilde{f})\right]^{T}[C]\left[\varepsilon\left(u^{n}\right)\right] \mu d z$

Note that the calculation of Eqs. (27) and (29) is performed using an analytical integration.

The introduction of the finite element approximation Eq. (23) in the variational Eq. (26) leads to the linear system
$\left[K_{z}(\tilde{f})\right]\left[q^{\nu}\right]=\left[\mathcal{R}_{v}\left(\tilde{f}, u^{n}\right)\right]$

where

- $\left[q^{v}\right]$ is the vector of the nodal displacements in the contravariant basis,

- $\left[K_{z}(\tilde{f})\right]$ is the stiffness matrix obtained by summing the elements' stiffness matrices $\left[K_{z}^{e}(\tilde{f})\right]=\int_{\Omega_{e}}\left[B_{\xi}\right]^{T}\left[k_{z}(\tilde{f})\right]\left[B_{\xi}\right] \sqrt{a} d \Omega_{e}$

- $\left[\mathcal{R}_{v}\left(\tilde{f}, u^{n}\right)\right]$ is the equilibrium residual obtained by summing the elements' residual load vectors $\left[\mathcal{R}_{v}^{e}\left(\tilde{f}, u^{n}\right)\right]=\int_{\Omega_{e}}\left[N_{\xi}\right]^{T}\left[t_{z}(\tilde{f})\right] \sqrt{a} d \Omega_{e}-\int_{\Omega_{e}}\left[B_{\xi}\right]^{T}\left[\sigma_{z}\left(\tilde{f}, u^{n}\right)\right] \sqrt{a} d \Omega_{e}$

\subsection{Finite element problem to be solved on $\Omega_{z}$}

For the sake of simplicity, the function $v^{(m+1)}$ which is assumed to be known, will be denoted $\tilde{v}$ (and $\tilde{V}$ ), and the function $f^{(m+1)}$ to be computed will be denoted $f$. The strain in Eq. (22) is defined in matrix notations as

$[\varepsilon(\tilde{V} f)]=\left[\sum_{\xi}(\tilde{v})\right]\left[\mathcal{E}_{f}\right]$

with

$\left[\Sigma_{\xi}(\tilde{v})\right]=\left[\begin{array}{cccccc}\tilde{v}_{1,1} / \mu & 0 & 0 & 0 & \tilde{v}_{3} /(\mu R) & 0 \\ 0 & 0 & \tilde{v}_{2,2} & 0 & 0 & 0 \\ 0 & 0 & 0 & 0 & 0 & \tilde{v}_{3} \\ 0 & 0 & 0 & \tilde{v}_{2} & \tilde{v}_{3,2} & 0 \\ -\tilde{v}_{1} /(\mu R) & \tilde{v}_{1} & 0 & 0 & \tilde{v}_{3,1} / \mu & 0 \\ \tilde{v}_{1,2} & 0 & \tilde{v}_{2,1} / \mu & 0 & 0 & 0\end{array}\right]$

The variational problem defined on $\Omega_{z}$ from Eq. (22) is

$$
\begin{aligned}
\int_{\Omega_{z}}\left[\delta \mathcal{E}_{f}\right]^{T}\left[k_{\xi}(\tilde{v})\right]\left[\mathcal{E}_{f}\right] \mu d z= & \left.\left([\delta f]^{T}\left[t_{\xi}(\tilde{v})\right] \mu\right)\right|_{z=z_{F}} \\
& -\int_{\Omega_{z}}\left[\delta \mathcal{E}_{f}\right]^{T}\left[\sigma_{\xi}\left(\tilde{v}, u^{n}\right)\right] \mu d z
\end{aligned}
$$

with

$$
\begin{aligned}
& {\left[k_{\xi}(\tilde{\mathcal{v}})\right]=\int_{\Omega}\left[\Sigma_{\xi}(\tilde{\mathcal{v}})\right]^{T}[C]\left[\Sigma_{\xi}(\tilde{\mathcal{v}})\right] \sqrt{a} d \Omega} \\
& {\left[t_{\xi}(\tilde{\mathcal{v}})\right]=\int_{\Omega}[\tilde{V}]^{T}[t] \sqrt{a} d \Omega} \\
& {\left[\sigma_{\xi}\left(\tilde{\mathcal{V}}, u^{n}\right)\right]=\int_{\Omega}\left[\Sigma_{\xi}(\tilde{\mathcal{V}})\right]^{T}[C]\left[\varepsilon\left(u^{n}\right)\right] \sqrt{a} d \Omega}
\end{aligned}
$$

The introduction of the finite element discretization Eq. (23) in the variational Eq. (33) leads to the linear system

$\left[K_{\xi}(\tilde{v})\right]\left[q^{f}\right]=\left[\mathcal{R}_{f}\left(\tilde{v}, u^{n}\right)\right]$

where $\left[q^{f}\right]$ is the vector of degree of freedom associated with the polynomial expansion in $\Omega_{z},\left[K_{\xi}(\tilde{v})\right]$ is a stiffness matrix defined by Eq. (38) and $\left[\mathcal{R}_{f}\left(\tilde{\nu}, u^{n}\right)\right]$ an equilibrium residual defined by Eq. (39)

$$
\begin{aligned}
& {\left[K_{\xi}(\tilde{\mathcal{v}})\right]=\int_{\Omega_{z}}\left[B_{z}\right]^{T}\left[k_{\xi}(\tilde{v})\right]\left[B_{z}\right] \mu d z} \\
& {\left[\mathcal{R}_{f}\left(\tilde{v}, u^{n}\right)\right]=\left.\left(\left[N_{z}\right]^{T}\left[t_{\xi}(\tilde{v})\right] \mu\right)\right|_{z=z_{F}}-\int_{\Omega_{z}}\left[B_{z}\right]^{T}\left[\sigma_{\xi}\left(\tilde{v}, u^{n}\right)\right] \mu d z}
\end{aligned}
$$

\section{Numerical results}

In this section, an eight-node quadrilateral FE based on the Serendipity interpolation functions is used for the unknowns depending on the in-plane coordinates. The geometry of the shell is 
approximated by this classical FE in the parametric space. The geometrical transformation is based on an explicit map $\vec{\Phi}$. A Gaussian numerical integration with $3 \times 3$ points is used to calculate the elementary matrices. Several static tests are presented validating our approach and evaluating its efficiency.

First, a convergence study is carried out to determine the suitable mesh for the further analysis. Then, the influence of the approximation on the factor $1 / \mu$ is addressed [43,1]. Three orders of expansion are considered. The influence of the numerical layers are also studied to show the possibility to refine the transverse description of the displacements and the stresses. Finally, the present approach is assessed for deep/shallow and thick/thin shells to show the wide range of validity.

All the following numerical assessments are based on the test case proposed by Ren [41]. It concerns a semi-infinite simply-supported cylindrical shell submitted to a sinusoidal pressure. It is described below:

Geometry: composite cross-ply cylindrical shell, $R=10, \phi \in\{\pi / 8, \pi / 3, \pi / 2\}$, with the following stacking sequences $\left[0^{\circ}\right],\left[0^{\circ} / 90^{\circ}\right],\left[0^{\circ} / 90^{\circ} / 0^{\circ}\right]$. All layers have the same thickness. $S=\frac{R}{e} \in\{2,4,10,40,100\}$. The panel is supposed infinite along the $x_{2}=\xi^{2}$ direction $\left(b_{\mathcal{C}}=8 a_{\mathcal{C}}\right.$ ) (Cf. Fig. 2).

Boundary conditions: simply-supported shell along its straight edges, subjected to a sinusoidal pressure along the curvature: $q\left(\xi^{1}\right)=q_{0} \sin \frac{\pi \xi^{1}}{R \phi}$.

Material properties: $E_{L}=25 \mathrm{GPa}, E_{T}=1 \mathrm{GPa}, G_{L T}=0.2 \mathrm{GPa}, G_{T T}=$ $0.5 \mathrm{GPa}, v_{L T}=v_{T T}=0.25$ where $L$ refers to the fiber direction, $T$ refers to the transverse direction.

Mesh: only a quarter of the structure is meshed. The mesh is constituted of $N_{x} \times N_{y}$ elements in the $\xi^{1}$ and $\xi^{2}$ directions respectively. A space ratio is considered in these two directions (ratio between the size of the larger and the smaller element). Numerical layers: $N_{z}$ is the total number of numerical layers.

Number of dofs: $N d o f_{x y}=3\left(N_{x}+1\right)\left(N_{y}+1\right)$ and $N d o f_{z}=12 \times$ $\alpha N C+3$ are the number of dofs of the two problems associated with $v_{j}^{i}$ and $f_{j}^{i}$ respectively. $\alpha$ is the number of numerical layers per physical layer. So the total number of dofs is $N d o f_{x y}+N d o f_{z}$.

Results: The results are made nondimensional using:

$$
\begin{gathered}
\bar{u}=u_{1}\left(0, b_{\mathcal{C}} / 2, z\right) \frac{E_{T}}{e q_{0} S^{3}}, \quad \bar{w}=u_{3}\left(a_{\mathcal{C}} / 2, b_{\mathcal{C}} / 2, z\right) \frac{100 E_{T}}{e q_{0} S^{4}}, \\
\bar{\sigma}_{\alpha \alpha}=\frac{\sigma_{\alpha \alpha}\left(a_{\mathcal{C}} / 2, b_{\mathcal{C}} / 2, z\right)}{q_{0} S^{2}}, \quad \bar{\sigma}_{13}=\frac{\sigma_{13}\left(0, b_{\mathcal{C}} / 2, z\right)}{q_{0} S}, \\
\bar{\sigma}_{33}=\frac{\sigma_{33}\left(a_{\mathcal{C}} / 2, b_{\mathcal{C}} / 2, z\right)}{q_{0}}
\end{gathered}
$$

Reference values: The 2D exact elasticity results are obtained as in [41].

Few iterations are required to reach the convergence of the fixed point algorithm (cf. Section 4.4). Moreover, for this test case, only one couple is needed to obtain the solution.

\subsection{Convergence study}

First, a convergence study is performed to obtain the suitable mesh on the most critical test, i.e. a one-layered shell with $S=100$. A constant space ratio for the mesh is taken as 10 for the $\xi^{1}$-direction and 50 in the $\xi^{2}$-direction. It is inferred from Table 1 that a $22 \times 10$ mesh (see Fig. 3 ) is adequate to model the structure. The results can be considered as converged values. The results issued from the Navier approach which is the asymptotic value of the present model, are also given. Moreover, the maximum error rate remains less than $0.9 \%$ for both displacements
Table 1

Convergence study - one layer $\left[0^{\circ}\right]-\mathrm{S}=100-\phi=\pi / 3-N_{z}=N C-$ mesh with space ratio (10/50).

\begin{tabular}{llllll}
\hline Mesh & $\bar{u}(0, e / 2)$ & $\bar{w}(L, 0)$ & $\bar{\sigma}_{11}(-e / 2)$ & $\bar{\sigma}_{13} \operatorname{Max}$ & $\bar{\sigma}_{33} \operatorname{Max}$ \\
\hline $6 \times 10$ & 20.1920 & 0.0631 & -0.6398 & 0.5568 & -16.310 \\
$12 \times 10$ & 23.8830 & 0.0746 & -0.7507 & 0.5717 & -18.456 \\
$22 \times 10$ & 24.1520 & 0.0755 & -0.7579 & 0.5679 & -18.517 \\
$32 \times 10$ & 24.1700 & 0.0755 & -0.7582 & 0.5668 & -18.501 \\
$64 \times 10$ & 24.1750 & 0.0755 & -0.7582 & 0.5658 & -18.483 \\
Navier & 24.1758 & 0.0755 & -0.7581 & 0.5652 & -18.349 \\
Exact & 24.1723 & 0.0755 & -0.7581 & 0.5651 & -18.346 \\
\hline
\end{tabular}

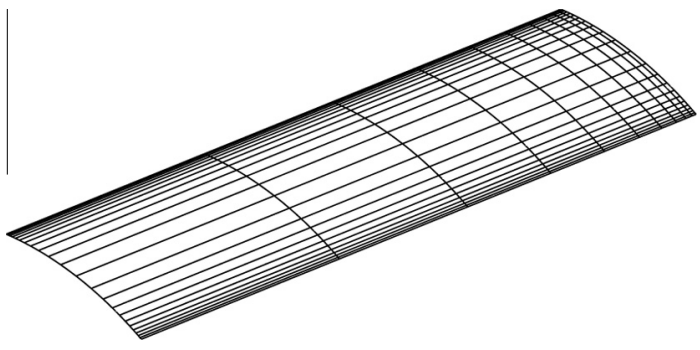

Fig. 3. Mesh $22 \times 10$ with a space ratio (10/50) for one quarter of the shell panel $\phi=\pi / 3-R=10$.

and stresses with respect to the 2D elasticity solution given by Ren [41]. This mesh is used for the following investigations.

\subsection{Influence of the expansion for the factor $1 / \mu$}

In this section, the influence of the approximation on the factor $1 / \mu=1 /(1+z / R)$ is addressed. The zero, one and two-order expansions are compared in Table 2 for very thick to thin homogeneous shells. The zero-order approximation (thin shell hypothesis or Love's hypothesis) seems to be valid only for the thin structure with $S \geqslant 40$. The solution is improved significantly for $S \leqslant 10$ when the order of the development of $1 / \mu$ increases. It concerns both the displacements and the stresses. Nevertheless, the use of the second-order expansion has a significant influence only for the thick shell.

It can be noticed that the error rate on the transverse shear and normal stresses remains high for the thick structures regardless of the degree of approximation of $1 / \mu$. This problem is investigated in the following section.

\subsection{Influence of the number of numerical layers}

The influence of the number of numerical layers is studied for the homogeneous case. In Table 3, four numerical layers per physical layer are considered for moderately thick to very thick cases that are the most representative ones. The results for two orders of expansion of $1 / \mu$ are given. First, it can be observed that the results are improved when compared with Table 2, regardless of the degree of approximation. In particular, the effect on the accuracy of the stresses is pronounced. Then, the use of the second order expansion of $1 / \mu$ drives to a very good agreement with the reference solution. The maximum error rate is $1 \%$, even for the very thick case. Nevertheless, this expansion is needed only for $S \leq 4$.

For further comparison, Fig. 4 shows the distribution of the transverse shear and normal stresses along the thickness $(S=2)$ for different number of numerical layers. Four numerical layers 
Table 2

influence of the order-approximation of $1 / \mu$ - one layer $\left[0^{\circ}\right]-\phi=\pi / 3-$ mesh $22 \times 10-N_{z}=N C$.

\begin{tabular}{|c|c|c|c|c|c|c|c|}
\hline$S$ & Expansion & Model & $\bar{u}(0, e / 2)$ & $\bar{w}(L, 0)$ & $\bar{\sigma}_{11}(-e / 2)$ & $\bar{\sigma}_{13} \operatorname{Max}$ & $\bar{\sigma}_{33} \operatorname{Max}$ \\
\hline \multirow[t]{7}{*}{2} & \multirow[t]{2}{*}{ Zero-order } & Present & 3.9745 & 0.7981 & -1.4083 & 0.4689 & 0.8867 \\
\hline & & Error & $16.67 \%$ & $19.84 \%$ & $42.63 \%$ & $15.73 \%$ & $11.33 \%$ \\
\hline & \multirow[t]{2}{*}{ One-order } & Present & 4.9847 & 1.0171 & -2.2567 & 0.6020 & 0.9345 \\
\hline & & Error & $4.51 \%$ & $2.17 \%$ & $8.06 \%$ & $8.19 \%$ & $6.55 \%$ \\
\hline & \multirow[t]{3}{*}{ Two-order } & Present & 4.7938 & 0.9767 & -2.1799 & 0.5781 & 0.8971 \\
\hline & & Error & $0.51 \%$ & $1.90 \%$ & $11.19 \%$ & $3.88 \%$ & $10.29 \%$ \\
\hline & & Exact & 4.7696 & 0.9956 & -2.4546 & 0.5565 & 1.0000 \\
\hline \multirow[t]{7}{*}{4} & \multirow[t]{2}{*}{ Zero-order } & Present & 2.3664 & 0.2792 & -1.0399 & 0.5289 & 0.9207 \\
\hline & & Error & $10.39 \%$ & $10.51 \%$ & $21.86 \%$ & $7.84 \%$ & $7.93 \%$ \\
\hline & \multirow[t]{2}{*}{ One-order } & Present & 2.6734 & 0.3153 & -1.3426 & 0.5990 & 0.9179 \\
\hline & & Error & $1.24 \%$ & $1.06 \%$ & $0.88 \%$ & $4.39 \%$ & $8.21 \%$ \\
\hline & \multirow[t]{3}{*}{ Two-order } & Present & 2.6386 & 0.3111 & -1.3253 & 0.5912 & 0.9058 \\
\hline & & Error & $0.09 \%$ & $0.26 \%$ & $0.42 \%$ & $3.02 \%$ & $9.42 \%$ \\
\hline & & Exact & 2.6408 & 0.3120 & -1.3309 & 0.5739 & 1.0000 \\
\hline \multirow[t]{7}{*}{10} & \multirow[t]{2}{*}{ Zero-order } & Present & 2.5622 & 0.1094 & -0.8064 & 0.5563 & -1.3617 \\
\hline & & Error & $4.55 \%$ & $4.56 \%$ & $9.37 \%$ & $4.05 \%$ & $9.58 \%$ \\
\hline & \multirow[t]{2}{*}{ One-order } & Present & 2.6930 & 0.1150 & -0.8928 & 0.5854 & -1.4172 \\
\hline & & Error & $0.32 \%$ & $0.32 \%$ & $0.33 \%$ & $0.97 \%$ & $5.89 \%$ \\
\hline & \multirow[t]{3}{*}{ Two-order } & Present & 2.6845 & 0.1146 & -0.8901 & 0.5835 & -1.4128 \\
\hline & & Error & $0.01 \%$ & $0.00 \%$ & $0.03 \%$ & $0.64 \%$ & $6.19 \%$ \\
\hline & & Exact & 2.6843 & 0.1146 & -0.8898 & 0.5798 & -1.5059 \\
\hline \multirow[t]{7}{*}{40} & \multirow[t]{2}{*}{ Zero-order } & Present & 9.2613 & 0.0770 & -0.7537 & 0.5625 & -7.0432 \\
\hline & & Error & $1.23 \%$ & $1.23 \%$ & $2.46 \%$ & $1.11 \%$ & $0.83 \%$ \\
\hline & \multirow[t]{2}{*}{ One-order } & Present & 9.3781 & 0.0780 & -0.7729 & 0.5698 & -7.1240 \\
\hline & & Error & $0.02 \%$ & $0.02 \%$ & $0.03 \%$ & $0.17 \%$ & $0.31 \%$ \\
\hline & \multirow[t]{3}{*}{ Two-order } & Present & 9.3756 & 0.0779 & -0.7727 & 0.5695 & -7.1233 \\
\hline & & Error & $0.01 \%$ & $0.01 \%$ & $0.00 \%$ & $0.12 \%$ & $0.30 \%$ \\
\hline & & Exact & 9.3765 & 0.0780 & -0.7727 & 0.5688 & -7.1021 \\
\hline \multirow[t]{7}{*}{100} & \multirow[t]{2}{*}{ Zero-order } & Present & 24.0330 & 0.0751 & -0.7504 & 0.5651 & -18.4420 \\
\hline & & Error & $0.58 \%$ & $0.58 \%$ & $1.02 \%$ & $0.00 \%$ & $0.52 \%$ \\
\hline & \multirow[t]{2}{*}{ One-order } & Present & 24.1530 & 0.0755 & -0.7579 & 0.5680 & -18.5150 \\
\hline & & Error & $0.08 \%$ & $0.08 \%$ & $0.03 \%$ & $0.52 \%$ & $0.92 \%$ \\
\hline & \multirow[t]{3}{*}{ Two-order } & Present & 24.1520 & 0.0755 & -0.7579 & 0.5679 & -18.5170 \\
\hline & & Error & $0.08 \%$ & $0.08 \%$ & $0.03 \%$ & $0.50 \%$ & $0.93 \%$ \\
\hline & & Exact & 24.1723 & 0.0755 & -0.7581 & 0.5651 & -18.3467 \\
\hline
\end{tabular}

Table 3

One layer $\left[0^{\circ}\right]-\phi=\pi / 3-$ mesh $22 \times 10-N_{z}=4 N C$.

\begin{tabular}{|c|c|c|c|c|c|c|c|}
\hline$S$ & Expansion & Model & $\bar{u}(0, e / 2)$ & $\bar{w}(L, 0)$ & $\bar{\sigma}_{11}(-e / 2)$ & $\bar{\sigma}_{13} \operatorname{Max}$ & $\bar{\sigma}_{33} \operatorname{Max}$ \\
\hline \multirow[t]{5}{*}{2} & \multirow[t]{2}{*}{ One-order } & Present & 4.9423 & 1.0326 & -2.5402 & 0.5755 & 1.0517 \\
\hline & & Error & $3.62 \%$ & $3.72 \%$ & $3.49 \%$ & $3.42 \%$ & $5.17 \%$ \\
\hline & \multirow[t]{3}{*}{ Two-order } & Present & 4.7640 & 0.9937 & -2.4596 & 0.5537 & 1.0118 \\
\hline & & Error & $0.12 \%$ & $0.19 \%$ & $0.20 \%$ & $0.49 \%$ & $1.18 \%$ \\
\hline & & Exact & 4.7696 & 0.9956 & -2.4546 & 0.5565 & 1.0000 \\
\hline \multirow[t]{5}{*}{4} & \multirow[t]{2}{*}{ One-order } & Present & 2.6754 & 0.3160 & -1.3491 & 0.5796 & 1.0229 \\
\hline & & Error & $1.31 \%$ & $1.28 \%$ & $1.37 \%$ & $1.00 \%$ & $2.29 \%$ \\
\hline & \multirow[t]{3}{*}{ Two-order } & present & 2.6411 & 0.3119 & -1.3321 & 0.5721 & 1.0097 \\
\hline & & Error & $0.01 \%$ & $0.03 \%$ & $0.09 \%$ & $0.31 \%$ & $0.97 \%$ \\
\hline & & Exact & 2.6408 & 0.3120 & -1.3309 & 0.5739 & 1.0000 \\
\hline \multirow[t]{5}{*}{10} & \multirow[t]{2}{*}{ One-order } & Present & 2.6930 & 0.1150 & -0.8926 & 0.5808 & -1.5205 \\
\hline & & Error & $0.32 \%$ & $0.32 \%$ & $0.31 \%$ & $0.17 \%$ & $0.97 \%$ \\
\hline & \multirow[t]{3}{*}{ Two-order } & Present & 2.6846 & 0.1146 & -0.8899 & 0.5789 & -1.5158 \\
\hline & & Error & $0.01 \%$ & $0.00 \%$ & $0.01 \%$ & $0.15 \%$ & $0.65 \%$ \\
\hline & & Exact & 2.6843 & 0.1146 & -0.8898 & 0.5798 & -1.5059 \\
\hline
\end{tabular}

Table 4

Two layers $\left[0^{\circ} / 90^{\circ}\right]-$ mesh $22 \times 10-N_{z}=N C-\phi=\pi / 3-$ two-order approximation of $1 / \mu$.

\begin{tabular}{|c|c|c|c|c|c|c|c|}
\hline$S$ & Model & $\bar{u}(0,-e / 2)$ & $\bar{w}(L, 0)$ & $\bar{\sigma}_{11}(-e / 2)$ & $\bar{\sigma}_{11}(e / 2)$ & $\bar{\sigma}_{13} \operatorname{Max}$ & $\bar{\sigma}_{33} \operatorname{Max}$ \\
\hline \multirow[t]{3}{*}{4} & Present & 12.6988 & 0.7041 & -2.7619 & 0.2724 & 0.9434 & 0.9975 \\
\hline & Error & $0.49 \%$ & $0.49 \%$ & $0.41 \%$ & $0.53 \%$ & $0.67 \%$ & $0.25 \%$ \\
\hline & Exact & 12.7610 & 0.7076 & -2.7732 & 0.2739 & 0.9371 & 1.0000 \\
\hline \multirow[t]{3}{*}{10} & Present & 17.7380 & 0.4436 & -2.3182 & 0.2339 & 0.9070 & -2.6116 \\
\hline & Error & $0.74 \%$ & $0.74 \%$ & $0.74 \%$ & $0.81 \%$ & $0.73 \%$ & $1.10 \%$ \\
\hline & Exact & 17.8707 & 0.4469 & -2.3356 & 0.2359 & 0.9136 & -2.6406 \\
\hline \multirow[t]{3}{*}{100} & Present & 135.3100 & 0.3974 & -2.1605 & 0.2315 & 0.8866 & -28.9150 \\
\hline & Error & $0.40 \%$ & $0.40 \%$ & $0.31 \%$ & $0.59 \%$ & $1.39 \%$ & $0.62 \%$ \\
\hline & Exact & 135.8586 & 0.3990 & -2.1672 & 0.2328 & 0.8745 & -28.7372 \\
\hline
\end{tabular}



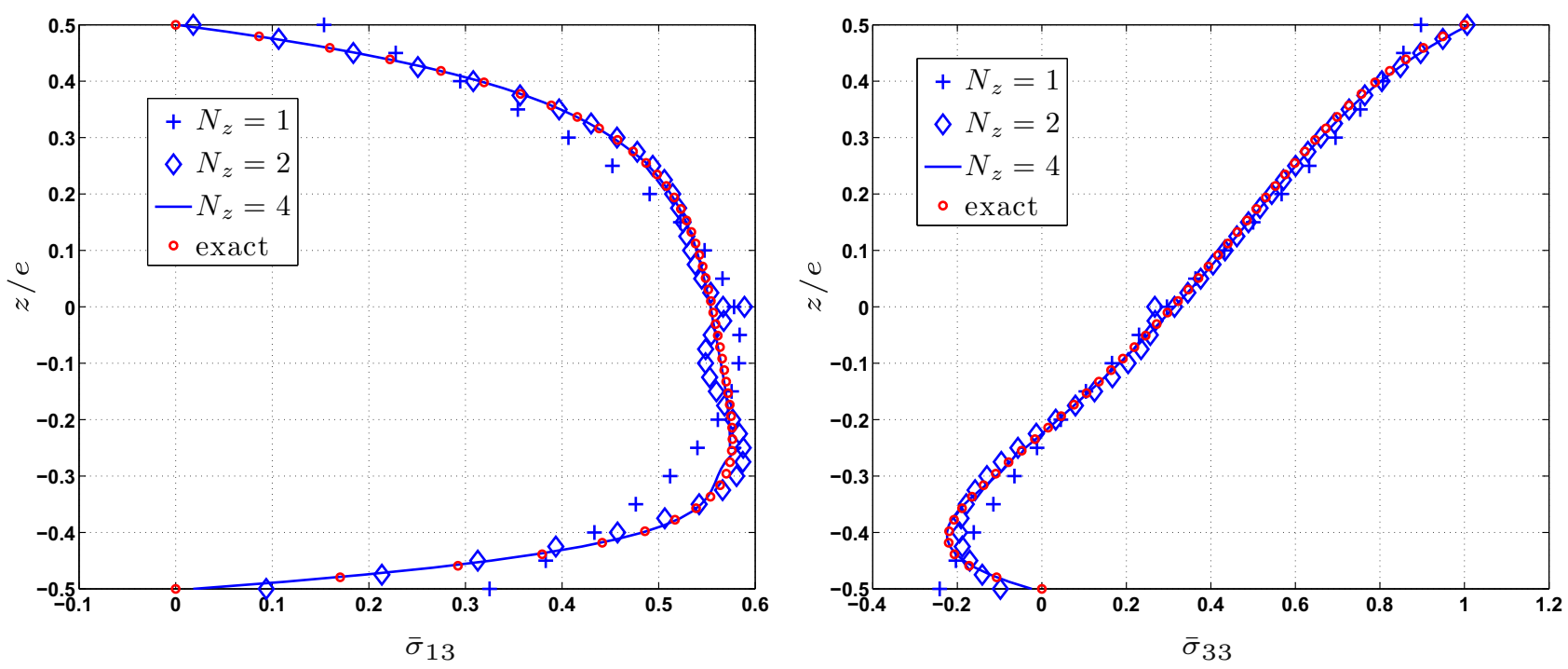

Fig. 4. Distribution of $\bar{\sigma}_{13}$ (left) and $\bar{\sigma}_{33}$ (right) along the thickness $-S=2$ - one layer $-N_{z}=\alpha N C, \alpha \in\{1,2,4\}-$ two-order approximation of $1 / \mu$.
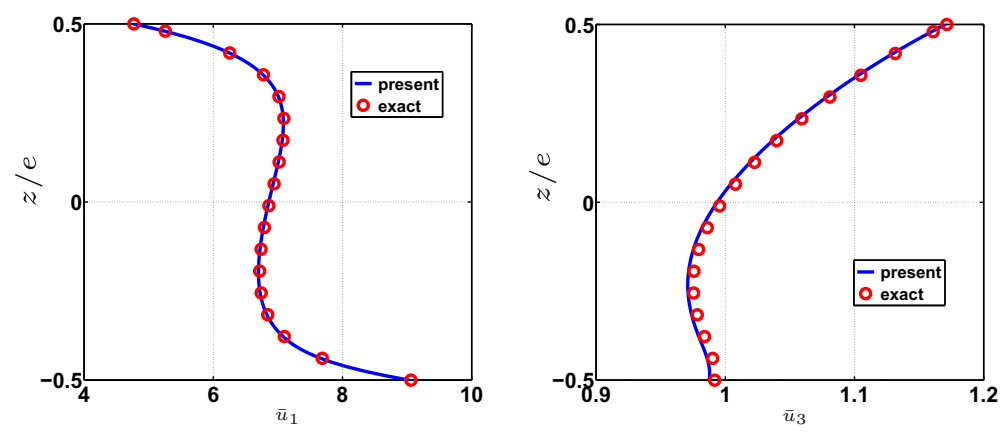

Fig. 5. Distribution of $\bar{u}_{1}$ (left), and $\bar{u}_{3}$ (right) along the thickness $-S=2-$ one layer $-N_{z}=4 N C$ - two-order approximation of $1 / \mu$.
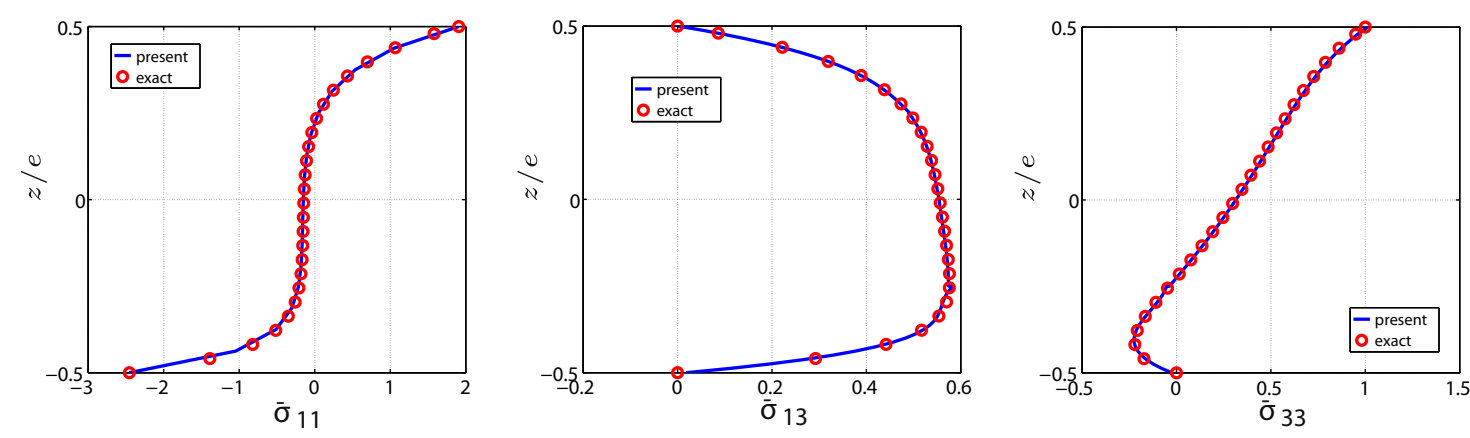

Fig. 6. Distribution of $\bar{\sigma}_{11}$ (left), $\bar{\sigma}_{13}$ (middle) and $\bar{\sigma}_{33}$ (right) along the thickness $-S=2-$ one layer $-N_{z}=4 N C-$ two-order approximation of $1 / \mu$.
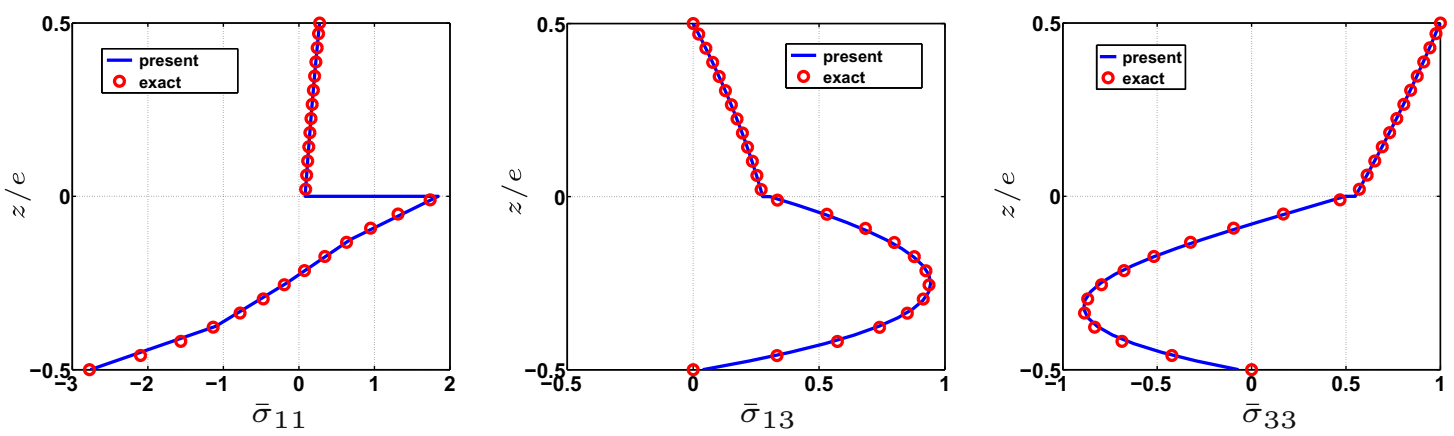

Fig. 7. Distribution of $\bar{\sigma}_{11}$ (left), $\bar{\sigma}_{13}$ (middle) and $\bar{\sigma}_{33}$ (right) along the thickness $-S=4-$ two layers $-\phi=\pi / 3$. 
Table 5

Three layers $\left[0^{\circ} / 90^{\circ} / 0^{\circ}\right]-$ mesh $22 \times 10-N_{z}=N C-\phi=\pi / 2$ - two-order approximation of $1 / \mu$.

\begin{tabular}{|c|c|c|c|c|c|c|}
\hline$S$ & Model & $\bar{u}(0, e / 2)$ & $\bar{w}(L, 0)$ & $\bar{\sigma}_{11}(-e / 2)$ & $\bar{\sigma}_{13}(0,0)$ & $\bar{\sigma}_{33} \operatorname{Max}$ \\
\hline \multirow[t]{3}{*}{4} & Present & 31.5706 & 1.9094 & -3.7645 & 0.9303 & -1.7613 \\
\hline & Error & $0.02 \%$ & $0.04 \%$ & $0.07 \%$ & $0.21 \%$ & $0.32 \%$ \\
\hline & Exact & 31.5782 & 1.9101 & -3.7620 & 0.9323 & -1.7556 \\
\hline \multirow[t]{3}{*}{10} & Present & 34.5670 & 0.7862 & -2.4574 & 0.9533 & -4.5981 \\
\hline & Error & $0.01 \%$ & $0.01 \%$ & $0.01 \%$ & $0.08 \%$ & $0.28 \%$ \\
\hline & Exact & 34.5699 & 0.7863 & -2.4572 & 0.9541 & -4.5854 \\
\hline \multirow[t]{3}{*}{100} & Present & 272.8200 & 0.5539 & -2.0900 & 0.9330 & -46.5030 \\
\hline & Error & $0.43 \%$ & $0.43 \%$ & $0.27 \%$ & $0.24 \%$ & $0.70 \%$ \\
\hline & Exact & 273.9914 & 0.5563 & -2.0956 & 0.9308 & -46.1786 \\
\hline
\end{tabular}

Table 6

three layers $\left[0^{\circ} / 90^{\circ} / 0^{\circ}\right]-$ mesh $22 \times 10-N_{z}=N C-\phi=\pi / 3-$ two-order approximation of $1 / \mu$.

\begin{tabular}{|c|c|c|c|c|c|c|}
\hline$S$ & Model & $\bar{u}(0, e / 2)$ & $\bar{w}(L, 0)$ & $\bar{\sigma}_{11}(-e / 2)$ & $\bar{\sigma}_{13}(0,0)$ & $\bar{\sigma}_{33} \mathrm{Max}$ \\
\hline \multirow[t]{3}{*}{4} & Present & 4.1668 & 0.4581 & -1.7737 & 0.4757 & 1.0052 \\
\hline & Error & $0.02 \%$ & $0.01 \%$ & $0.12 \%$ & $0.17 \%$ & $0.52 \%$ \\
\hline & Exact & 4.1658 & 0.4581 & -1.7715 & 0.4765 & 1.0000 \\
\hline \multirow[t]{3}{*}{10} & Present & 3.5370 & 0.1440 & -0.9951 & 0.5251 & -1.4917 \\
\hline & Error & $0.01 \%$ & $0.00 \%$ & $0.02 \%$ & $0.07 \%$ & $0.25 \%$ \\
\hline & exact & 3.5367 & 0.1440 & -0.9949 & 0.5254 & -1.4879 \\
\hline \multirow[t]{3}{*}{100} & Present & 25.1330 & 0.0785 & -0.7865 & 0.5243 & -17.2160 \\
\hline & Error & $0.05 \%$ & $0.05 \%$ & $0.00 \%$ & $0.17 \%$ & $0.45 \%$ \\
\hline & Exact & 25.1468 & 0.0786 & -0.7866 & 0.5234 & -17.1396 \\
\hline
\end{tabular}

Table 7

Three layers $\left[0^{\circ} / 90^{\circ} / 0^{\circ}\right]-$ mesh $22 \times 10-N_{z}=N C-\phi=\pi / 8-$ two-order approximation of $1 / \mu$.

\begin{tabular}{|c|c|c|c|c|c|c|}
\hline$S$ & Model & $\bar{u}(0,-e / 2)$ & $\bar{w}(L, 0)$ & $\bar{\sigma}_{11}(-e / 2)$ & $\bar{\sigma}_{13}(0,0)$ & $\bar{\sigma}_{33} \operatorname{Max}$ \\
\hline \multirow[t]{3}{*}{4} & Present & 0.3168 & 0.0343 & -0.3827 & 0.1074 & 0.9987 \\
\hline & Error & $0.21 \%$ & $0.05 \%$ & $0.18 \%$ & $0.38 \%$ & $0.13 \%$ \\
\hline & Exact & 0.3175 & 0.0343 & -0.3834 & 0.1078 & 1.0000 \\
\hline \multirow[t]{3}{*}{10} & Present & 0.1887 & 0.0076 & -0.1996 & 0.1488 & 1.0014 \\
\hline & Error & $0.01 \%$ & $0.02 \%$ & $0.01 \%$ & $0.08 \%$ & $0.14 \%$ \\
\hline & Exact & 0.1887 & 0.0076 & -0.1996 & 0.1489 & 1.0000 \\
\hline \multirow[t]{3}{*}{100} & Present & 0.2158 & 0.0013 & -0.1009 & 0.1769 & -1.9355 \\
\hline & Error & $0.01 \%$ & $0.01 \%$ & $0.01 \%$ & $0.02 \%$ & $0.12 \%$ \\
\hline & Exact & 0.2158 & 0.0013 & -0.1009 & 0.1768 & -1.9332 \\
\hline
\end{tabular}
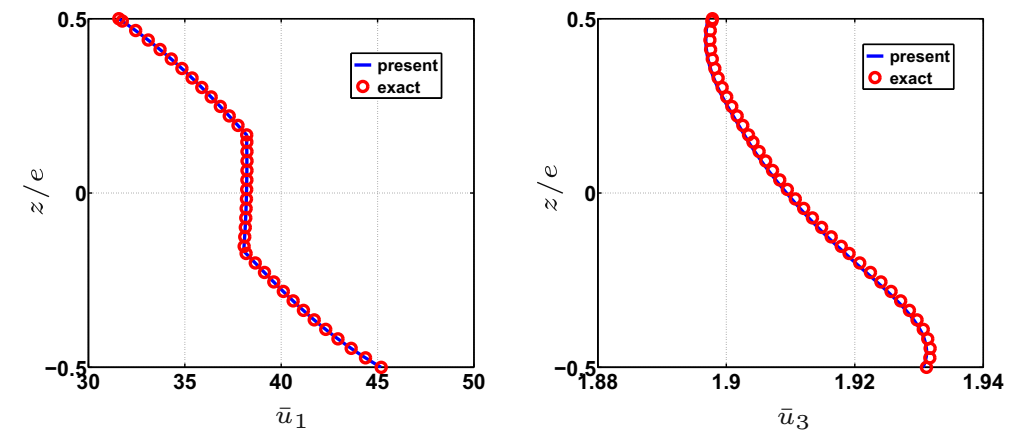

Fig. 8. Distribution of $\bar{u}_{1}$ (left), and $\bar{u}_{3}$ (right) along the thickness $-S=4-$ three layers $-\phi=\pi / 2-$ two-order approximation of $1 / \mu$.

are sufficient to recover the reference solution. The boundary conditions on the upper and lower surfaces are nearly fulfilled. To show the accuracy of the approach, both displacements and stresses are represented on Figs. 5 and 6. Complex distributions along the thickness can be obtained. We can observe that the dissymmetry of the results is well-captured.
It should be also noted that only the number of dofs of the 1D problem increases $\left(\mathrm{Ndof}_{z}=12 \times \alpha \mathrm{NC}+3\right)$, so the additional computational cost is negligible, the size of the 2D problem remaining unchanged. That is different for a LW approach where the total number of unknowns would be $\operatorname{Ndof}_{x y} \times N d o f_{z}$ $=N d o f_{x y} \times(12 \times \alpha N C+3)$. 

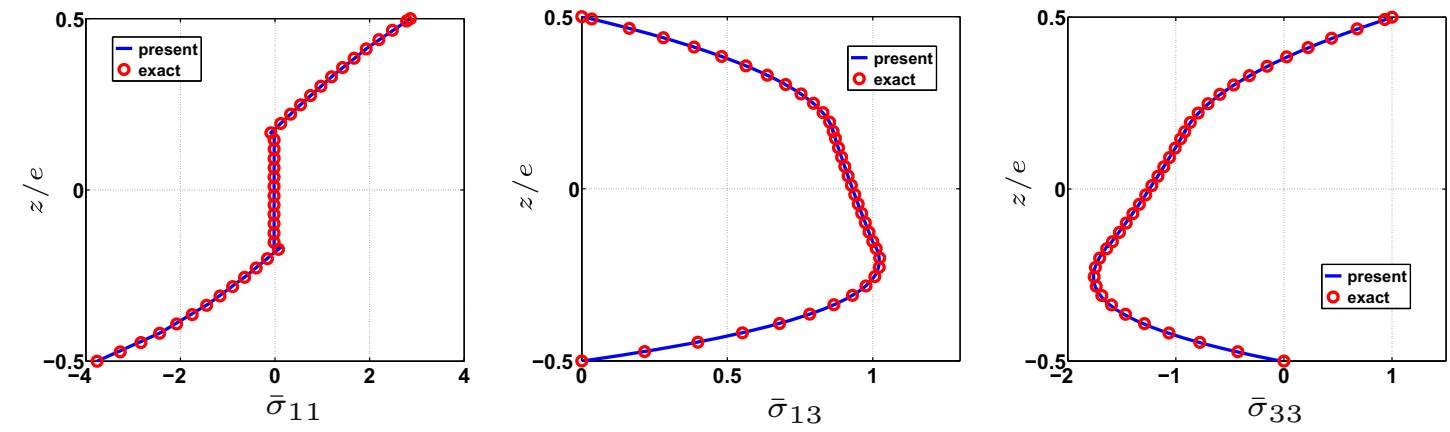

Fig. 9. Distribution of $\bar{\sigma}_{11}$ (left), $\bar{\sigma}_{13}$ (middle) and $\bar{\sigma}_{33}$ (right) along the thickness $-S=4$ - three layers $-\phi=\pi / 2-$ two-order approximation of $1 / \mu$.

\subsection{Anti-symmetric shell}

In this section, the approach is assessed on an anti-symmetric shell, that is a two-layer case $\left[0^{\circ} / 90^{\circ}\right.$. It is inferred from Table 4 that the accuracy of the results is very good. The error rate is less than $1.3 \%$ regardless of the slenderness ratio. Only the in-plane, transverse shear and normal stresses are shown in Fig. 7 for a thick structure. The present approach yields very satisfactory results for the anti-symmetric shell.

\subsection{Deep/shallow shell}

This section deals with the capability of the present approach to model both deep and shallow shells. For this purpose, three opening angles are considered, $\phi=\pi / 8, \pi / 3, \pi / 2$ for a three-layer shell. A second-order expansion of $1 / \mu$ is considered as thick to thin structures are involved in this study. Only one numerical layer is considered. Table 5-7 show the excellent accuracy of the results for all the configurations. The error rate is less than $0.7 \%$. The variation of the normalized in-plane, transverse shear and normal stresses, and inplane, transverse displacements through the thickness for $S=4$ are presented in Figs. 8 and 9 for $\phi=\pi / 2$. The results are in very good agreement with the reference solution of the deep shell.

\section{Conclusion}

In this article, the PGD is applied to the modeling of cylindrical composite shell using a fourth-order LW description of the displacements. The results are assessed through different classical benchmarks. The influence of classical strain assumption (approximation of $1 / \mu$ ) is discussed. We have shown the importance of the expansion of this term for thick shells. The accuracy of the results could be also improved by using numerical layers in each physical layer without increasing significantly the computational cost. Finally, accurate results have been obtained for a wide type of shell configurations. Both deep/shallow and very thick/thin shell structures can be modeled with an excellent accuracy. Moreover, the present method allows to address complex layered structures without resorting to a costly Layer-Wise FE model.

Based on these promising results, the membrane and shear locking phenomenon will be addressed in future investigation.

\section{References}

[1] Dau F, Polit O, Touratier M. A efficient $c^{1}$ finite element with continuity requirements for multilayered/sandwich shell structures. Comput Struct 2004;82:1889-99.

[2] Zienkiewicz O, Taylor R. The finite element method, 5th ed., vol. 2. Butterworth-Heinemann; 2000.
[3] Vidal P, D’Ottavio M, Thaier MB, Polit O. An efficient finite shell element for the static response of piezoelectric laminates. J Intell Mater Syst Struct 2011;22(7):671-90. http://dx.doi.org/10.1177/1045389X11402863.

[4] Reddy J. Mechanics of laminated composite plates and shells - theory and analysis. CRC Press Inc.; 2004.

[5] Jeyachandrabose C, Kirkhope J. Explicit formulation of two anisotropic, triangular, thin, shallow shell elements. Comput Struct 1987;25:415-36.

[6] Rao K. A rectangular laminated anisotropic shallow thin shell finite element. Comput Methods Appl Mech Eng 1978;15:13-33.

[7] Qatu M, Leissa A. Bending analysis of laminated plates and shells by different methods. Comput Struct 1994;52:529-39.

[8] Hossain S, Sinha P, Sheikh A. A finite element formulation for the analysis of laminated composite shells. Comput Struct 2004;82:1623-38.

[9] Leissa A. Vibration of shells, NASA SP-288, Nasa report; 1973.

[10] Sgambitterra G, Adumitroaie A, Barbero E, Tessler A. A robust three-node shell element for laminated composites with matrix damage. Compos Part B: Eng J 2011;42:41-50.

[11] Kant T, Menon M. Estimation of interlaminar stresses in fibre reinforced composite cylindrical shells. Comput Struct 1991;38:131-47.

[12] Balah M, Al-Ghamedy H. Finite element formulation of a third order laminated finite rotation shell element. Comput Struct 2002;80:1975-90.

[13] Cinefra M, Carrera E. Shell finite elements with different through-thethickness kinematics for the linear analysis of cylindrical multilayered structures. Int J Non-Newtonian Fluid Mech 2013;93(2):160-82.

[14] Brank B. On composite shell models with a piecewise linear warping function. Compos Struct 2003;59:163-71.

[15] Bhaskar K, Varadan T. A higher-order theory for bending analysis of laminated shells of revolution. Comput Struct 1991;40(4):815-9.

[16] Seide P, Chaudhuri R. Triangular finite element for analysis of thick laminated shells. Int J Numer Methods Eng 1987;24(8):1563-79.

[17] Klinkel S, Gruttmann F, Wagner W. A continuum based three dimensional shell element for laminated structures. Comput Struct 1999;71:43-62.

[18] Botello S, Onate E, Canet J. A layer-wise triangle for analysis of laminated composite plates and shells. Comput Struct 1999;70:635-46.

[19] Liu M, To C. Free vibration analysis of laminated composite shell structures using hybrid strain based layerwise finite elements. Finite Elem Anal Des 2003;40:83-120.

[20] Baser Y, Ding Y. Interlaminar stress analysis of composites: layer-wise shell finite elements including transverse strains. Comput Eng 1995;5(5): 485-99.

[21] Vu-Quoc L, Tan X. Efficient hybrid-EAS solid element for accurate stress prediction in thick laminated beams, plates, and shells. Comput Methods Appl Mech Eng 2013;253:337-55.

[22] Yasin M, Kapuria S. An efficient layerwise finite element for shallow composite and sandwich shells. Compos Struct 2013;98:202-14.

[23] Shariyat M. Non-linear dynamic thermo-mechanical buckling analysis of the imperfect laminated and sandwich cylindrical shells based on a global-local theory inherently suitable for non-linear analyses. Int J Non-Linear Mech 2011;46:253-71.

[24] Kapania R. A review on the analysis of laminated shells. J Pressure Vessel Technol 1989;111:88-96.

[25] Noor A, Burton W. Assessment of computational models for multilayered composite shells. Appl Mech Rev 1990;43(4):67-97.

[26] Gilewski W, Radwanska M. A survey of finite element models for the analysis of moderately thick shells. Finite Elem Anal Des 1991;9:1-21.

[27] Yang H, Saigal S, Masud A, Kapania R. A survey of recent shell finite element. Int J Numer Methods Eng 2000;47:101-27.

[28] Carrera E. Theories and finite elements for multilayered, anisotropic composite plates and shells. Arch Comput Methods Eng 2002;9(2):87-140.

[29] Reddy J, Arciniega R. Shear deformation plate and shell theories: from Stavsky to present. Mech Adv Mater Struct 2004;11:535-82.

[30] Hohe J, Librescu L. Advances in the structural modeling of elastic sandwich panels. Mech Adv Mater Struct 2004;11(4-5):395-424.

[31] Qatu M, Asadi E, Wang W. Review of recent literature on static analyses of composite shells: 2000-2010. Open J Compos Mater 2012;2:61-86. 
[32] Chinesta F, Ammar A, Leygue A, Keunings R. An overview of the proper generalized decomposition with applications in computational rheology. J Non-Newtonian Fluid Mech 2011;166(11):578-92.

[33] Chinesta F, Ammar A, Cueto E. Recent advances and new challenges in the use of the proper generalized decomposition for solving multidimensional models. Arch Comput Methods Eng 2010;17(4):327-50.

[34] Ammar A, Mokdada B, Chinesta F, Keunings R. A new family of solvers for some classes of multidimensional partial differential equations encountered in kinetic theory modeling of complex fluids. J Non-Newtonian Fluid Mech 2006; 139:153-76.

[35] Allix O, Vidal P. A new multi-solution approach suitable for structural identification problems. Comput Methods Appl Mech Eng 2002;191(25 26):2727-58.

[36] Savoia M, Reddy J. A variational approach to three-dimensional elasticity solutions of laminated composite plates. J Appl Mech ASME 1992;59:166-75.

[37] Bognet B, Bordeu F, Chinesta F, Leygue A, Poitou A. Advanced simulation of models defined in plate geometries: $3 \mathrm{~d}$ solutions with $2 \mathrm{~d}$ computational complexity. Comput Methods Appl Mech Eng 2012;201-204:1-12. http:/ dx.doi.org/10.1016/i.cma.2011.08.025.

[38] Vidal P, Gallimard L, Polit O. Assessment of a composite beam finite element based on the proper generalized decomposition. Compos Struct 2012;94(5):1900-10. http://dx.doi.org/10.1016/j.compstruct.2011.12.016.

[39] Vidal P, Gallimard L, Polit O. Composite beam finite element based on the proper generalized decomposition. Comput Struct 2012;102-103:76-86. http://dx.doi.org/10.1016/i.compstruc.2012.03.008.

[40] Vidal P, Gallimard L, Polit O. Proper generalized decomposition and layer-wise approach for the modeling of composite plate structures. Int J Solids Struct 2013;50(14-15):2239-50. http://dx.doi.org/10.1016/j.ijsolstr.2013.03.034.

[41] Ren JG. Exact solutions for laminated cylindrical shells in cylindrical bending. Comput Sci Tech 1987;29:169-87.

[42] Bernadou M. Finite element methods for thin shell problems. John Wiley et Sons; 1996.

[43] Icardi U, Ruotolo R. Laminated shell model with second-order expansion of the reciprocals of lamé coefficicents $h_{\alpha}, h_{\beta}$ and interlayer continuities fulfilment. Compos Struct 2002;56:293-313. 